\title{
Complete Bubble Destruction is Essential for Quantitative Assessment From the Replenishment Curve in Real-time Myocardial Contrast Echocardiography
}

\author{
Kentaro Otani, MS, Kasumi Masuda, MS, Toshihiko Asanuma, MD, \\ Ayako Hongawa, BS, Miyako Takaoka, MS, Takashi Uehara, MS, \\ Fuminobu Ishikura, MD and Shintaro Beppu, MD \\ Division of Functional Diagnostic Science, Graduate School of Medicine, \\ Osaka University, Suita, Japan
}

\begin{abstract}
Background. Myocardial perfusion can be quantified using the replenishment curve of the myocardial opacification derived after transient high power ultrasound exposure (burst) in real-time myocardial contrast echocardiography (MCE). However, the effect of acoustic power of burst for bubble destruction may confound results. Thus, the goal of the present study was to examine the effect of burst intensity on the parameters of the replenishment curve.

Methods. Myocardial opacification of the left ventricular short-axis view was observed using SIEMENS Sequoia 512 (mechanical index $=0.1$ ) during infusion of Definity in eight open-chest dogs. The mechanical index (MI) of burst was set as high and low $(0$ and $-11 \mathrm{~dB})$, and the regions of interest were placed on the anterior and lateral walls of the left ventricle. The temporal changes in video intensity of the end-diastolic phase after the burst procedure were fitted to an exponential function: $y=a\left(1-e^{-\beta t}\right)+c$. The plateau video intensity was defined as the A-value which was calculated as the sum of the a and $c$ values.

Results. The A-value remained constant with changes in the burst MI. Although the video intensity after the high intensity $(\mathrm{OdB})$ burst was similar to that of the baseline, the video intensity after the low intensity $(-11 \mathrm{~dB})$ burst was significantly higher than that of the baseline (anterior: $5.5 \pm 2.2 \mathrm{~dB}$ at baseline vs. $7.4 \pm 2.4 \mathrm{~dB}$ at $-11 \mathrm{~dB}$ burst; lateral: $5.9 \pm 2.5 \mathrm{~dB}$ at baseline vs. $9.3 \pm 3.2 \mathrm{~dB}$ at $-11 \mathrm{~dB}$ burst), which suggests that low $\mathrm{Ml}$ burst results in incomplete bubble destruction. Furthermore, the $\beta$-value increased as the burst MI decreased (anterior: $0.39 \pm 0.07$ and $0.54 \pm 0.15$; lateral: $0.27 \pm 0.05$ and $0.39 \pm 0.12$ in response to a burst $\mathrm{Ml}$ of 0 and $-11 \mathrm{~dB}$, respectively).

Conclusions. The myocardial microbubble velocity ( $\beta$-value) derived from the replenishment curve by using bubble destruction technique in real-time MCE is overestimated when bubble destruction is incomplete.
\end{abstract}

(J Echocardiogr 2005; 3: 21-26)

Key words: ultrasound, contrast echocardiography, coronary microcirculation

\section{Introduction}

In 1998, Wei et al. described a novel approach of assessing myocardial perfusion using the replenishment curve as determined by myocardial contrast

Received January 25, 2005; revision received March 9, 2005; accepted March 17, 2005

Address for correspondence: Shintaro Beppu, MD

Division of Functional Diagnostic Science, Graduate School of Medicine, Osaka University,

1-7 Yamadaoka, Suita 565-0871, Japan.

Telephone: +81-6-6879-2561

Fax: +81-6-6879-2561

E-mail: beppu@sahs.med.osaka-u.ac.jp

(C) 2003 Journal of Echocardiography echocardiography (MCE) [1]. Since that time, many investigators have validated this method for diagnosis of coronary stenosis and myocardial ischemia [2-6].

The concept of the replenishment curve derived from MCE requires complete destruction of microbubbles inside the myocardium by a single high acoustic power exposure in intermittent triggered $\mathrm{MCE}$ and by the several high acoustic power pulses (burst) in realtime MCE. However, Bahlmann et al. [7] demonstrated that microbubbles are not completely destroyed by the 1-frame exposure of high intensity ultrasound in intermittent triggered MCE or by the consecutive 10 high power pulses during real-time MCE. These data indicate that microbubbles may persist within the 
myocardium after the burst procedure, which may affect the assessment of a replenishment curve during real-time MCE. Thus the goal of the present study was to determine the effect of changes in microbubble destruction by various burst intensities on the parameters of the replenishment curve.

\section{Methods}

\section{Animal Preparation.}

All protocols were approved by our institutional animal care and use committee and were in compliance with institutional guidelines for the care and use of laboratory animals.

Eight healthy dogs $(14.2 \pm 0.8 \mathrm{~kg})$ were anesthetized with intravenous pentobarbital sodium $(35 \mathrm{mg} / \mathrm{kg})$, followed by pentobarbital sodium infusion ( 6 to $8 \mathrm{mg} / \mathrm{kg}$ per hour) and saline $(1 \mathrm{ml} / \mathrm{min})$ during the experiment. Animals were intubated with an endotracheal tube and ventilated with a Harvard-type respirator (Model SN-480-3, Shinano Manufacturing Co. Ltd., Tokyo, Japan) using room air. A left lateral thoracotomy was performed at the fifth intercostal space, and the heart was suspended in a pericardial cradle.

\section{MCE.}

Definity ${ }^{\circledR}$ (Bristol-Myers Squibb Medical Imaging, Billerica, Mass) was administered continuously (0.075 $\mathrm{ml} / \mathrm{min}$ ) via a peripheral intravenous catheter placed in the forearm. Myocardial opacification was examined at the short-axis view at the mid-papillary muscle

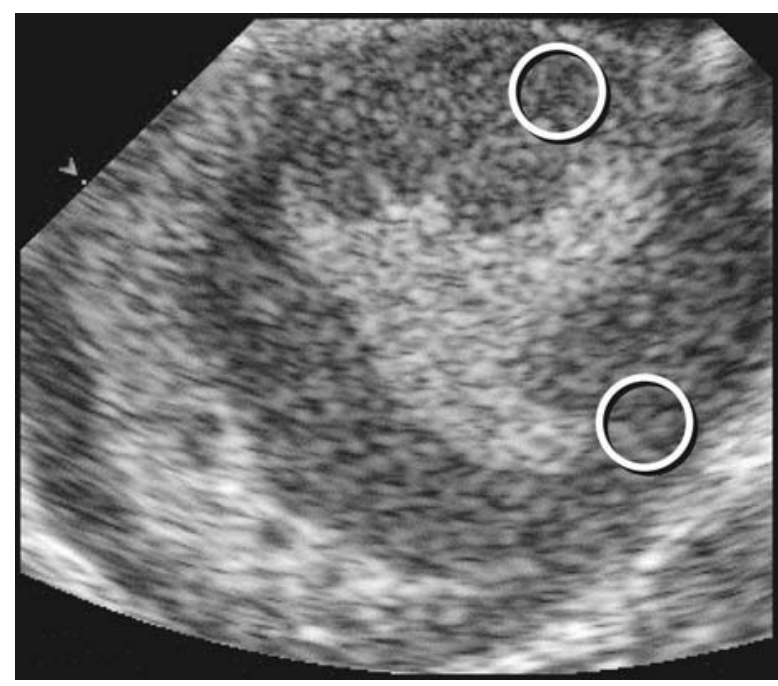

Fig. 1. Setting of regions of interest (ROIs). The transmural ROls were set on the anterior wall and lateral wall of the left ventricle. level of the left ventricle using the coherent contrast imaging method of Sequoia 512 (SIEMENS Medical Solutions USA, Inc., Mountain View, CA) and a 3V2c transducer. The transmitted and received frequencies were set as 1.75 and $3.5 \mathrm{MHz}$, respectively. An acoustic interface with a thickness of $1.0 \mathrm{~cm}$ (SonarAID, Geistlich-Pharma AG, Wolhusen, Switzerland) was set between the transducer and the anterior surface of the heart, and the transducer position was fixed with a hand-made clamp. Transducer focus was set at the center of the left ventricular cavity, and the dynamic range was set at $50 \mathrm{~dB}$. The overall gain setting and image depth were optimized at the beginning of the experiment and kept constant throughout the experiment.

Myocardial opacification may vary with the cardiac cycle. Despite the use of low mechanical index $(\mathrm{MI}=0.1)$, intermittent triggered MCE was adopted at end-diastole or at end-systole in this study. Time of burst was also triggered at the same cardiac phase and 5 times exposure was adopted for the burst procedure. The acoustic intensity of the burst was set at $0 \mathrm{~dB}$ $(\mathrm{MI}=1.6)$ for complete and $-11 \mathrm{~dB}(\mathrm{MI}=0.52)$ for incomplete bubble disruption. The consecutive MCE images were digitally captured before and after the burst procedure. Circular regions of interest (ROIs) with $0.8 \mathrm{~mm}$ diameters were placed on the anterior and lateral walls of the left ventricle (Figure 1), and those

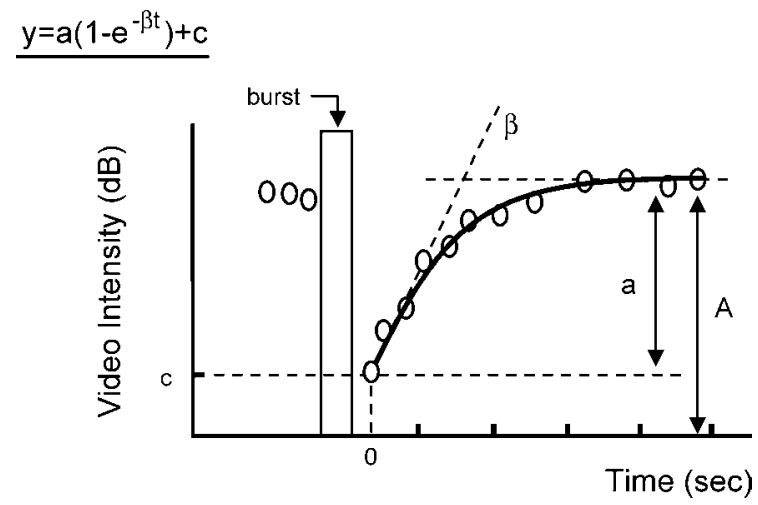

Fig. 2. Fitting function scheme. The first end-diastolic or end-systolic phase after the burst procedure was defined as Time 0 , and the time course of the myocardial opacification was fitted to an exponential function: $y=a\left(1-e^{-\beta t}\right)+c$. The c-value was set as the video intensity just after the burst procedure. The plateau video intensity (A-value) was calculated as the sum of the $a$ and $c$ values. 
positions were adjusted manually to correct for the translational and rotational motion of the heart. Video intensity of each ROI was measured in decibel (dB) units by an off-line computer (Data Pro, Noesis, Courtaboeuf, France).

The time course of video intensity after the burst procedure was fitted to an exponential function, $\mathrm{y}=\mathrm{a}\left(1-\mathrm{e}^{-\beta \mathrm{t}}\right)+\mathrm{c}$, using commercially available software (Origin 6.0j, Microcal, North Hampton, Mass). The aand $\beta$-values represent the increment of the video intensity and the rate of rise of the video intensity, respectively. The first frame after the burst procedure was defined as Time 0 , and the c-value was the video intensity just after burst procedure. The plateau video intensity (A-value) was calculated as the sum of the a and $\mathrm{c}$ values (Figure 2).

\section{Statistical analysis.}

Data are represented as mean $\pm \mathrm{SD}$. The comparison of the video intensity between baseline (before contrast infusion) and just after the burst procedure and replenishment curve parameters generated by high and low intensity bursts were performed by
Paired t-test. A P value less than 0.05 was considered statistically significant for all comparisons.

\section{Results}

Myocardial opacification just after the burst procedure depended on the burst intensity. Figure 3 shows a representative case triggered at the end-diastolic phase. The video intensity was lower just after the 0 $\mathrm{dB}$ burst than after the $-11 \mathrm{~dB}$ burst (Figure $3 \mathrm{~A}$ ), although the video intensities at the plateau phase were nearly similar regardless of changes in the burst intensity (Figure 3B).

The video intensities at baseline and after the burst procedure are shown in Figure 4. The video intensities at baseline and after the high intensity $(0 \mathrm{~dB})$ burst were similar (anterior: $5.5 \pm 2.2 \mathrm{~dB}$ at baseline vs. $5.4 \pm 2.9 \mathrm{~dB}$ at $0 \mathrm{~dB}$ burst; lateral: $5.9 \pm 2.5 \mathrm{~dB}$ at baseline vs. $7.0 \pm 3.1 \mathrm{~dB}$ at $0 \mathrm{~dB}$ burst) in end-diastolic triggering. However, the video intensity just after low intensity $(-11 \mathrm{~dB})$ burst was significantly higher than that of the baseline (anterior: $5.5 \pm 2.2 \mathrm{~dB}$ at baseline vs. $7.4 \pm 2.4 \mathrm{~dB}$ at $-11 \mathrm{~dB}$ burst; lateral: $5.9 \pm 2.5 \mathrm{~dB}$ at baseline vs.

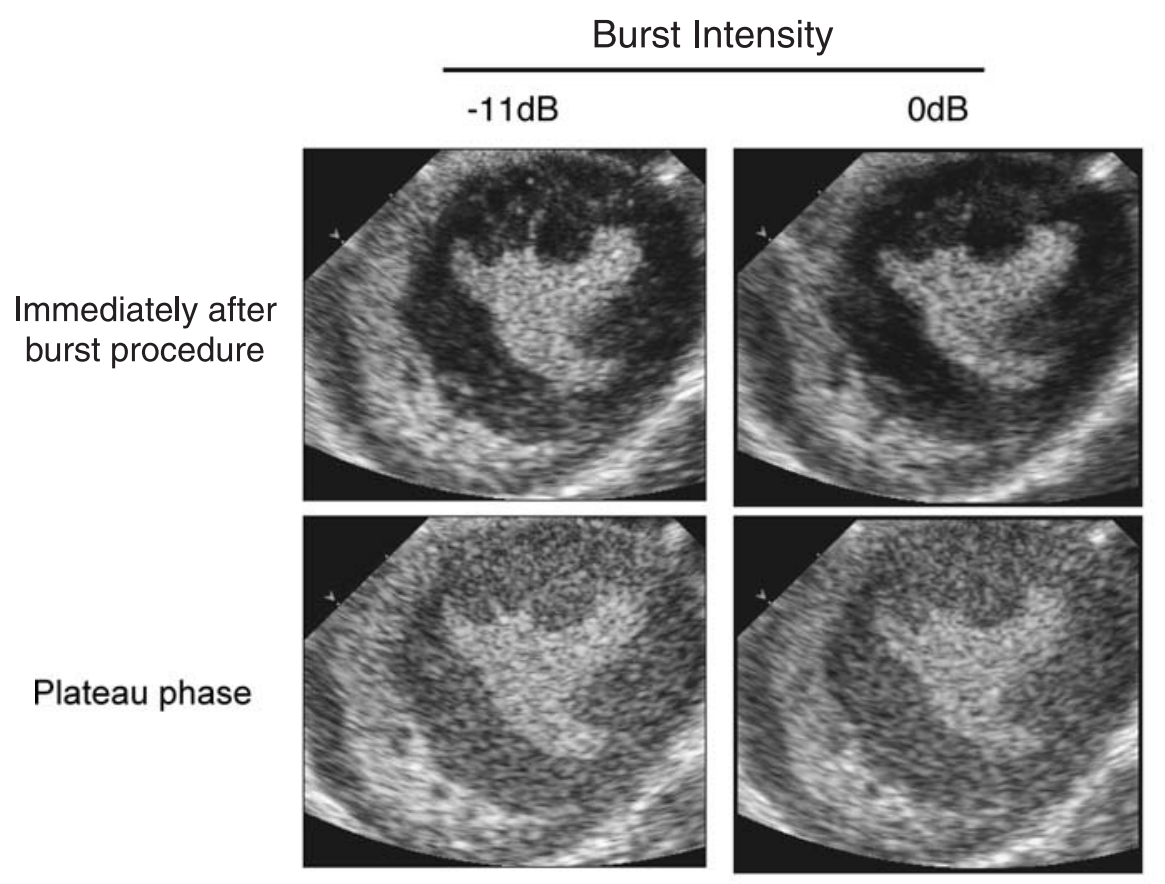

Fig. 3. Representative case of contrast echocardiograms triggered at end-diastolic phase just after the burst procedure and at the plateau phase. The video intensity was higher just after the low $\mathrm{MI}(-11 \mathrm{~dB})$ burst than after the high $\mathrm{Ml}(\mathrm{O} \mathrm{dB})$ burst. However, the video intensities at the plateau phase were nearly similar irrespective of the acoustic intensity of burst. 


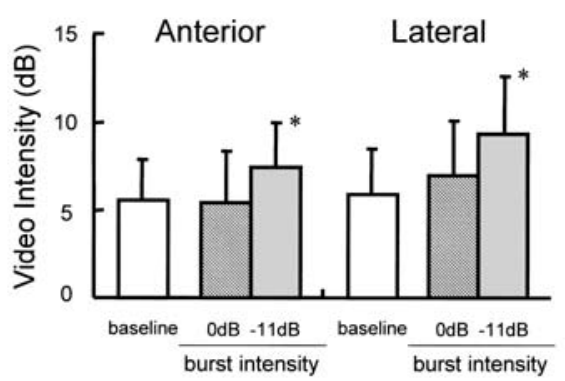

End-diastolic triggering

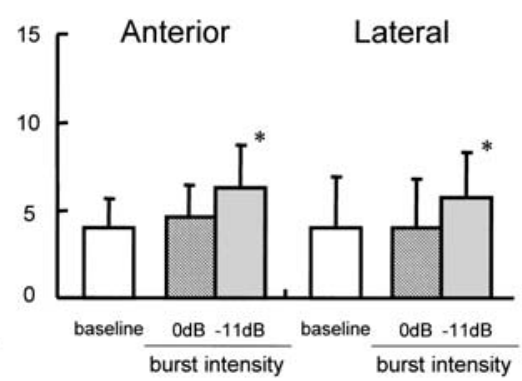

End-systolic triggering

Fig. 4. The video intensity at baseline and immediately after the burst procedure. The video intensity immediately after the low intensity burst was significantly higher than that of baseline in both end-diastolic triggering and end-systolic triggering. $*$ :p $<0.05$ vs. baseline.

Table 1. Parameters of the replenishment curve derived from different burst intensities

\begin{tabular}{lllll}
\hline & \multicolumn{2}{c}{ Anterior } & \multicolumn{2}{c}{ Lateral } \\
\hline End-diastolic triggering & $0 \mathrm{~dB}$ burst & $-11 \mathrm{~dB}$ burst & $0 \mathrm{~dB}$ burst & $-11 \mathrm{~dB}$ burst \\
\hline A-value $(\mathrm{dB})$ & $20.8 \pm 2.6$ & $20.9 \pm 2.2$ & $20.5 \pm 3.3$ & $20.9 \pm 2.5$ \\
$\beta$-value & $0.39 \pm 0.07$ & $0.54 \pm 0.15^{*}$ & $0.27 \pm 0.05^{\dagger}$ & $0.39 \pm 0.12^{*}$ \\
\hline End-systolic triggering & & & \\
\hline A-value $(\mathrm{dB})$ & $18.9 \pm 2.0$ & $18.9 \pm 1.6$ & $18.2 \pm 2.8$ & $18.3 \pm 2.7$ \\
$\beta$-value & $0.41 \pm 0.11$ & $0.51 \pm 0.14^{*}$ & $0.32 \pm 0.07$ & $0.45 \pm 0.11^{*}$ \\
\hline
\end{tabular}

${ }^{*} \mathrm{p}<0.05$ vs. $0 \mathrm{~dB}$ burst

$\dagger_{\mathrm{p}}<0.05$ vs. anterior with $0 \mathrm{~dB}$ burst

$9.3 \pm 3.2 \mathrm{~dB}$ at $-11 \mathrm{~dB}$ burst) in end-diastolic triggering (Figure 4A). Furthermore, in Figure 4B, similar results were observed in the end-systolic triggering (anterior: $4.0 \pm 1.7 \mathrm{~dB}, 4.6 \pm 1.7 \mathrm{~dB}$ and $6.2 \pm 2.3 \mathrm{~dB}$; lateral: $4.0 \pm 2.8 \mathrm{~dB}, 4.0 \pm 2.7 \mathrm{~dB}$ and $5.7 \pm 2.4 \mathrm{~dB}$ at baseline and at burst intensity of $0 \mathrm{~dB}$ and $-11 \mathrm{~dB}$, respectively).

Table 1 shows the parameters of the replenishment curve. The A-value was almost identical irrespective of the acoustic intensity of burst in both the anterior and lateral wall in end-diastolic triggering (anterior: $20.8 \pm 2.6 \mathrm{~dB}$ vs. $20.9 \pm 2.2 \mathrm{~dB}$, lateral: $20.5 \pm 3.3 \mathrm{~dB}$ vs. $20.9 \pm 2.5 \mathrm{~dB}$ by burst intensity of $0 \mathrm{~dB}$ and $-11 \mathrm{~dB}$, respectively) and end-systolic triggering (anterior: $18.9 \pm 2.0 \mathrm{~dB}$ vs. $18.9 \pm 1.6 \mathrm{~dB}$, lateral: $18.2 \pm 2.8 \mathrm{~dB}$ vs.18.3 $\pm 2.7 \mathrm{~dB}$ by burst intensity of $0 \mathrm{~dB}$ and $-11 \mathrm{~dB}$, respectively). However, the $\beta$-value with the low intensity burst was significantly higher than that with high intensity burst both in end-diastolic triggering (anterior: $0.39 \pm 0.07$ vs. $0.54 \pm 0.15$, lateral: $0.27 \pm 0.05$ vs. $0.39 \pm 0.12$ by burst intensity of $0 \mathrm{~dB}$ and $-11 \mathrm{~dB}$, respectively) and in end-systolic triggering (anterior: $0.41 \pm 0.11$ vs. $0.51 \pm 0.14$, lateral: $0.32 \pm 0.07$ vs. $0.45 \pm 0.11$, respectively).

Representative replenishment curves after complete and incomplete bubble destruction are shown in Figure 5. Although the shapes of the replenishment curves were identical when comparing $0 \mathrm{~dB}$ and $-11 \mathrm{~dB}$, the time to plateau intensity of a replenishment curve derived after $-11 \mathrm{~dB}$ burst was faster than that derived after $0 \mathrm{~dB}$ burst. The $\beta$-value was higher by the $-11 \mathrm{~dB}$ burst than by the $0 \mathrm{~dB}$ burst ( 0.56 vs. 0.32 ). 


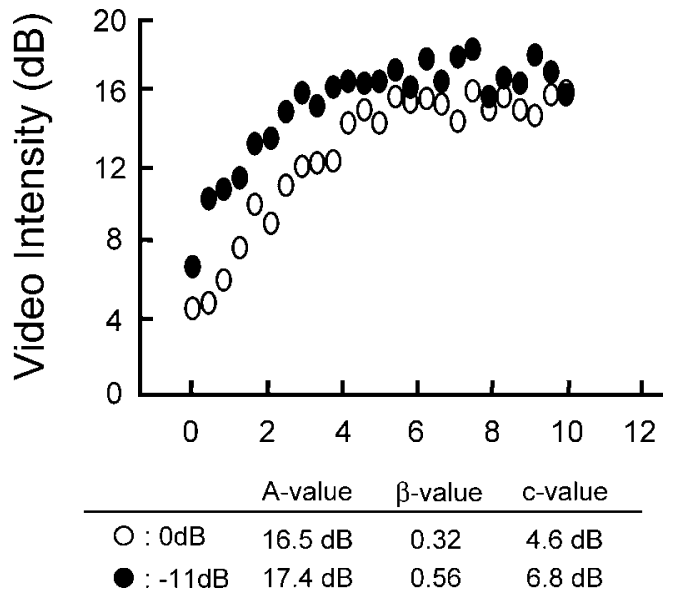

Fig. 5. Replenishment curves by low (closed circle) and high intensity burst (open circle). Although the shapes of the two replenishment curves were similar, the $\beta$-value was larger with the low intensity burst than that with the high intensity burst.

\section{Discussion}

Bahlmann et al. reported that the microbubbles were not destroyed completely by the 1 -frame high power ultrasound exposure during intermittent triggered MCE or by the 10 frames of high power ultrasound exposure during real-time MCE [7]. Furthermore, acoustic power is lower at deeper and lateral regions inside the acoustic field in the electrical scanning. Thus, the present study hypothesized that the degree of microbubble destruction by the high power ultrasound exposure (burst) would be different when comparing different regions within the same burst intensity.

\section{Low-MI intermittent triggered MCE.}

The simultaneous observation of cardiac function and myocardial perfusion is the chief advantage of realtime MCE compared with other modalities. Further, assessment of wall motion is of utility for the diagnosis of coronary artery disease. In contrast, the low-MI intermittent triggered MCE is of better utility in examining the effect of burst intensity on the quantitative parameters of myocardial perfusion. For example, the absence of cardiac cyclic motion and the need for only minimal correction of the ROIs makes the analysis of video intensity relatively easier with low-MI intermittent triggered MCE. Further, this technique does not require selection of the specific cardiac phase that is needed for real-time echocardiograms. Thus, the present study utilized low-MI intermittent triggered MCE for assessment of myocardial perfusion.

\section{Effect of burst intensity.}

Several factors contribute to the changes in the $\beta$ value in response to incomplete bubble destruction after the low intensity burst (Table 1). First, the $\beta$ value is defined as the inverse of the time to plateau. Therefore, the $\beta$-value should be increased when the video intensity at the onset of the replenishment curve was not low enough at Time 0 in response to incomplete bubble destruction. Second, the degree of microbubble destruction and the width of bubble destruction would vary when comparing different burst intensities even within the same myocardial region. Hence, the width of bubble destruction might be wider after a high intensity burst than after a low intensity burst.

As shown in Table 1, persistent microbubbles alter parameters of the replenishment curve in end-diastolic triggering and in end-systolic triggering. This suggests that the effect of the persistent microbubbles on the replenishment curve parameters occurs regardless of cardiac phase.

\section{Other factors influencing on replenishment curve parameters.}

As shown in Table 1 , the $\beta$-value was smaller in the lateral wall than in the anterior wall. Lafitte et al. [8] and Köster et al. [9] demonstrated that replenishment curve parameters varied with different focal settings or depth in the acoustic field. Thus, regional differences in the $\beta$-value may be attributed to the difference in depth and the position inside the scan field.

Swales et al. [10] demonstrated that the $\beta$-value decreased due to the destruction of microbubbles inside the left ventricular cavity, which are the resource of the bubbles flowing into the coronary arteries, in response to excess high power ultrasound exposure. The effect of persistence of microbubbles after the burst procedure and the excess bubble destruction on the replenishment curve parameters would be critical when replenishment curve parameters are compared before and after pharmacological or exercise stress. Consequently, the novel approach for compensating their effects would be needed.

\section{Clinical implications.}

Visual assessment of replenishment kinetics may provide useful information for the diagnosis of coro- 
nary artery disease, even if the bubbles are not destroyed completely at the "burst" procedure. However, the bubbles should be destroyed completely when the quantitative assessment is required. Hence, adjustments of burst intensity and its duration may be important factors when making comparisons between regions of differing depth.

$\mathrm{Yu}$ et al. recently characterized the parametric images of myocardial perfusion using low-MI intermittent triggered MCE [11]. These parametric and colorcoded images may be of utility for clinical determinations of the extent of the myocardial perfusion defect and the abnormal perfusion area. Further study of the effect of regional heterogeneity of bubble destruction on the parametric images of myocardial perfusion would be beneficial.

\section{Conclusions}

The parameter of replenishment curve, especially the $\beta$-value, was overestimated when bubbles were destroyed incompletely in real-time MCE. This undesirable effect might mislead the diagnosis of coronary stenosis in real-time MCE with the pharmacological or exercise stress test. Complete bubble destruction should be required to assess the myocardial perfusion accurately in real-time MCE.

\section{References}

1. Wei K, Jayaweera AR, Firoozan S, Linka A, Skyba DM, Kaul S. Quantification of myocardial blood flow with ultrasound-induced destruction of microbubbles administered as a constant venous infusion. Circulation 1998; 97: 47383.

2. Masugata H, Lafitte S, Peters B, Strachan GM, DeMaria AN. Comparison of real-time and intermittent triggered myocardial contrast echocardiography for quantification of coronary stenosis severity and transmural perfusion gradient. Circulation 2001; 104: 1550-1556.

3. Masugata H, Peters B, Lafitte S, Strachan GM, Ohmori
K, DeMaria AN. Quantitative assessment of myocardial perfusion during graded coronary stenosis by real-time myocardial contrast echo refilling curves. J Am Coll Cardiol 2001; 37: 262-9.

4. Leong-Poi H, Le E, Rim SJ, Sakuma T, Kaul S, Wei K. Quantification of myocardial perfusion and determination of coronary stenosis severity during hyperemia using real-time myocardial contrast echocardiography. J Am Soc Echocardiogr 2001; 14: 1173-82.

5. Lafitte S, Masugata H, Peters B, et al. Comparative value of dobutamine and adenosine stress in the detection of coronary stenosis with myocardial contrast echocardiography. Circulation 2001; 103: 2724-2730.

6. Otani K, Toshida T, Iwata A, Asanuma T, Ishikura F, Beppu S. Adenosine triphosphate stress myocardial contrast echocardiography detects coronary artery stenosis with greater sensitivity than wall motion abnormality. J Am Soc Echocardiogr 2004; 17: 1275-80.

7. Bahlmann EB, McQuillan BM, Handschumacher MD, et al. Effect of destructive pulse duration on the detection of myocardial perfusion in myocardial contrast echocardiography: in vitro and in vivo observations. J Am Soc Echocardiogr 2002; 15: 1440-7.

8. Lafitte S, Masugata H, Peters B, et al. Accuracy and reproducibility of coronary flow rate assessment by realtime contrast echocardiography: In vitro and in vivo studies. J Am Soc Echocardiogr 2001; 14: 1010-9.

9. Köster J, Schlosser T, Pohl C, et al. Blood flow assessment by ultrasound-induced destruction of echocontrast agents using harmonic power Doppler imaging. Echocardiography 2001; 18: 1-8.

10. Swales J, Leong-Poi H, Fisher N, Bin JP, Kaul S, Lindner JR. Does destruction of microbubbles in the LV cavity influence microbubble replenishment rate? J Am Soc Echocardiogr 2001; 14: 438 [abstract].

11. Yu EHC, Skyba DM, Leong-Poi H, et al. Incremental value of parametric quantitative assessment of myocardial perfusion by triggered low-power myocardial contrast echocardiography. J Am Coll Cardiol 2004; 43: 180713. 